\title{
Lower bounds for non-relativistic atomic energies
}

Robbie T. Ireland ${ }^{\dagger}$ Peter Jeszenszki and Edit Mátyus , ${ }^{*, \ddagger}$ Rocco Martinazzo, ${ }^{*, \Phi}$ and Miklos Ronto and Eli Pollak*,§

$\dagger$ †nstitute of Chemistry, Eötvös Loránd University, Pázmány Péter sétány 1/A, Budapest, H-1117, Hungary,

and School of Chemistry, University of Glasgow, University Avenue, G12 8QQ, Glasgow, United Kingdom

$\ddagger$ Institute of Chemistry, Eötvös Loránd University, 1117 Budapest, Hungary

IDepartment of Chemistry, University of Milan, Milan,

and Institute of Molecular Science and Technologies (ISTM), Consiglio Nazionale delle

Ricerche (CNR), Milan, Italy

$\S$ Chemical and Biological Physics Department, Weizmann Institute of Science, 76100,

Rehovot, Israel

E-mail: edit.matyus@ttk.elte.hu; rocco.martinazzo@unimi.it; eli.pollak@weizmann.ac.il 


\section{Supporting Information}

\section{Derivation of Matrix Elements}

The $\left\langle\frac{1}{r_{i j} r_{p a}}\right\rangle$ expectation value, corresponding to the product of the electron-electron and electron-nuclei potential energy operators, can be solved by beginning with Eq. 3.1,

$$
\psi(\boldsymbol{r}, \boldsymbol{\sigma})=\mathcal{A}\{\phi(\boldsymbol{r}, \boldsymbol{A}) \chi(\boldsymbol{\sigma}, \theta)\}
$$

The spin functions are constructed by coupling elementary spin functions to the selected total spin quantum numbers. As such, it is the spatial ECG functions $\phi(\boldsymbol{r}, \boldsymbol{A})$ that are of interest when deriving the expectation value, where the $k$ th ECG basis function is written as

$$
\phi_{k}(\boldsymbol{r}, \boldsymbol{A})=\exp \left[-\boldsymbol{r}^{T}\left(\boldsymbol{A}_{k} \otimes \boldsymbol{I}_{3}\right) \boldsymbol{r}\right]
$$

$\boldsymbol{r} \in \mathbb{R}^{3 n_{\mathrm{p}}}$ is the vector containing the Cartesian positions of the $n_{\mathrm{p}}$ particles, $\boldsymbol{A}_{k} \in \mathbb{R}^{n_{\mathrm{p}} \times n_{\mathrm{p}}}$ is the positive-definite symmetric matrix containing the non-linear parameters of the $k$ th ECG basis function and $\boldsymbol{I}_{3} \in \mathbb{R}^{3 \times 3}$ is the identity matrix. Then, the $k$ th, $l$ th element takes the form

$$
\left\langle\phi_{k}\left|\frac{1}{r_{i j} r_{p a}}\right| \phi_{l}\right\rangle=\int_{-\infty}^{+\infty} \mathrm{d} \boldsymbol{r} \frac{1}{r_{i j} r_{p a}} \exp \left[-\boldsymbol{r}^{T} \underline{\boldsymbol{A}}_{k l} \boldsymbol{r}\right]
$$

where we have defined $\underline{\boldsymbol{A}}_{k l}=\left(\boldsymbol{A}_{k} \otimes \boldsymbol{I}_{3}\right)+\left(\boldsymbol{A}_{l} \otimes \boldsymbol{I}_{3}\right)$. By using the following Gaussian integral transforms,

$$
\frac{1}{r_{i j}}=\frac{2}{\sqrt{\pi}} \int_{0}^{+\infty} \mathrm{d} t \exp \left[-\boldsymbol{r}^{T} \underline{\boldsymbol{J}}_{i j} \boldsymbol{r} t^{2}\right]
$$




$$
\frac{1}{r_{p a}}=\frac{2}{\sqrt{\pi}} \int_{0}^{+\infty} \mathrm{d} u \exp \left[-\boldsymbol{r}^{T} \underline{\boldsymbol{J}}_{p a} \boldsymbol{r} u^{2}\right]
$$

we arrive at

$$
\left\langle\phi_{k}\left|\frac{1}{r_{i j} r_{p a}}\right| \phi_{l}\right\rangle=\frac{4}{\pi} \int_{0}^{+\infty} \mathrm{d} t \int_{0}^{+\infty} \mathrm{d} u \int_{-\infty}^{+\infty} \mathrm{d} \boldsymbol{r} \exp \left[-\boldsymbol{r}^{T}\left(\underline{\boldsymbol{A}}_{k l}+t^{2} \underline{\boldsymbol{J}}_{i j}+u^{2} \underline{\boldsymbol{J}}_{p a}\right) \boldsymbol{r}\right],
$$

where $\boldsymbol{J}_{m n} \in \mathbb{R}^{n_{\mathrm{p}} \times n_{\mathrm{p}}}$ is a rank-1 matrix with element

$$
\left(\boldsymbol{J}_{m n}\right)_{\alpha \beta}=\delta_{m \alpha} \delta_{m \beta}+\delta_{n \alpha} \delta_{n \beta}-\delta_{m \alpha} \delta_{n \beta}-\delta_{n \alpha} \delta_{m \beta}
$$

Furthermore, we define $\underline{\boldsymbol{J}}_{m n}=\boldsymbol{J}_{m n} \otimes \boldsymbol{I}_{3}$. Using the Gaussian integral expression

$$
\int_{-\infty}^{+\infty} \mathrm{d} \boldsymbol{r} \exp \left[-\boldsymbol{r}^{T} \boldsymbol{M} \boldsymbol{r}\right]=\frac{\pi^{\frac{3 n}{2}}}{\left|\boldsymbol{M}^{\frac{1}{2}}\right|}
$$

for $n$-electrons, we obtain

$$
\left\langle\phi_{k}\left|\frac{1}{r_{i j} r_{p a}}\right| \phi_{l}\right\rangle=\frac{4}{\pi} \pi^{\frac{3 n}{2}} \int_{0}^{+\infty} \mathrm{d} t \int_{0}^{+\infty} \mathrm{d} u\left|\boldsymbol{A}_{k l}+t^{2} \boldsymbol{J}_{i j}+u^{2} \boldsymbol{J}_{p a}\right|^{-\frac{3}{2}}
$$

The determinant above can be rewritten an

$$
\left|\boldsymbol{A}_{k l}+t^{2} \boldsymbol{J}_{i j}+u^{2} \boldsymbol{J}_{p a}\right|^{-\frac{3}{2}}=\left|\boldsymbol{I}+t^{2} \boldsymbol{J}_{i j} \boldsymbol{A}_{k l}^{-1}+u^{2} \boldsymbol{J}_{p a} \boldsymbol{A}_{k l}^{-1}\right|^{-\frac{3}{2}}\left|\boldsymbol{A}_{k l}\right|^{-\frac{3}{2}}
$$

meaning we can use the following rank-2 determinant expression,

$$
\begin{aligned}
& \left|\boldsymbol{G}+\boldsymbol{H}_{1}+\boldsymbol{H}_{2}\right| \\
= & |\boldsymbol{G}|\left[1+\operatorname{Tr}\left\{\boldsymbol{H}_{1} \boldsymbol{G}^{-1}\right\}+\operatorname{Tr}\left\{\boldsymbol{H}_{2} \boldsymbol{G}^{-1}\right\}+\operatorname{Tr}\left\{\boldsymbol{H}_{1} \boldsymbol{G}^{-1}\right\} \operatorname{Tr}\left\{\boldsymbol{H}_{2} \boldsymbol{G}^{-1}\right\}-\operatorname{Tr}\left\{\boldsymbol{H}_{1} \boldsymbol{G}^{-1} \boldsymbol{H}_{2} \boldsymbol{G}^{-1}\right\}\right]
\end{aligned}
$$

where $\operatorname{rank}\left(\boldsymbol{H}_{1}\right)=\operatorname{rank}\left(\boldsymbol{H}_{2}\right)=1$. 
For matrices $\boldsymbol{A}$ and $\boldsymbol{B}$, it can be shown that $\operatorname{rank}(\boldsymbol{A} \boldsymbol{B})=\operatorname{rank}(\boldsymbol{A})$, if $\boldsymbol{B}$ is a non-singular matrix. As such, $\operatorname{rank}\left(\boldsymbol{J}_{i j} \boldsymbol{A}_{k l}^{-1}\right)=\operatorname{rank}\left(\boldsymbol{J}_{i j}\right)$. As each column of $\boldsymbol{J}_{i j}$ is a linear combination of the first, it is a rank-1 matrix, meaning that the $\boldsymbol{J}_{i j} \boldsymbol{A}_{k l}^{-1}$ matrix is also rank-1. The same is true for $\boldsymbol{J}_{p a} \boldsymbol{A}_{k l}^{-1}$.

By letting $\boldsymbol{H}_{1}=t^{2} \boldsymbol{J}_{i j} \boldsymbol{A}_{k l}^{-1}$ and $\boldsymbol{H}_{2}=u^{2} \boldsymbol{J}_{p a} \boldsymbol{A}_{k l}^{-1}$, the expectation value then reads

$$
\left\langle\phi_{k}\left|\frac{1}{r_{i j} r_{p a}}\right| \phi_{l}\right\rangle=\frac{4}{\pi} \pi^{\frac{3 n}{2}}\left|\boldsymbol{A}_{k l}\right|^{-\frac{3}{2}} \int_{0}^{+\infty} \mathrm{d} t \int_{0}^{+\infty} \mathrm{d} u\left[1+t^{2} a+u^{2} b+u^{2} t^{2}(a b-c)\right]^{-\frac{3}{2}},
$$

where we have defined

$$
\begin{aligned}
& a=\operatorname{Tr}\left\{\boldsymbol{J}_{i j} \boldsymbol{A}_{k l}^{-1}\right\}, \\
& b=\operatorname{Tr}\left\{\boldsymbol{J}_{p a} \boldsymbol{A}_{k l}^{-1}\right\}, \\
& c=\operatorname{Tr}\left\{\boldsymbol{J}_{i j} \boldsymbol{A}_{k l}^{-1} \boldsymbol{J}_{p a} \boldsymbol{A}_{k l}^{-1}\right\} .
\end{aligned}
$$

We can then see that

$$
\begin{aligned}
1+t^{2} a+u^{2} b+u^{2} t^{2}(a b-c) & =1+t^{2} a+u^{2} b+u^{2} b t^{2} a-u^{2} t^{2} c \\
& =\left(1+t^{2} a\right)\left(1+u^{2} b-u^{2} \frac{t^{2} c}{1+t^{2} a}\right) \\
& =\left(1+t^{2} a\right)\left[1+u^{2}\left(b-\frac{t^{2} c}{1+t^{2} a}\right)\right],
\end{aligned}
$$

which gives

$$
\left\langle\phi_{k}\left|\frac{1}{r_{i j} r_{p a}}\right| \phi_{l}\right\rangle=\frac{4}{\pi} \pi^{\frac{3 n}{2}}\left|\boldsymbol{A}_{k l}\right|^{-\frac{3}{2}} \int_{0}^{+\infty} \mathrm{d} t \int_{0}^{+\infty} \mathrm{d} u\left(1+t^{2} a\right)^{-\frac{3}{2}}\left[1+u^{2}\left(b-\frac{t^{2} c}{1+t^{2} a}\right)\right]^{-\frac{3}{2}} .
$$


The following substitution can then be made,

$$
w=\frac{u^{2}}{1+u^{2}\left(b-\frac{t^{2} c}{1+t^{2} a}\right)},
$$

leading to

$$
\begin{aligned}
\left\langle\phi_{k}\left|\frac{1}{r_{i j} r_{p a}}\right| \phi_{l}\right\rangle & =\frac{2}{\pi} \pi^{\frac{3 n}{2}}\left|\boldsymbol{A}_{k l}\right|^{-\frac{3}{2}} \int_{0}^{+\infty} \mathrm{d} t \int_{0}^{\left(b-\frac{t^{2} c}{1+t^{2} a}\right)^{-1}} \mathrm{~d} w\left(1+t^{2} a\right)^{-\frac{3}{2}} \frac{1}{w^{\frac{1}{2}}} \\
& =\frac{4}{\pi} \pi^{\frac{3 n}{2}}\left|\boldsymbol{A}_{k l}\right|^{-\frac{3}{2}} \int_{0}^{+\infty} \mathrm{d} t\left(1+t^{2} a\right)^{-\frac{3}{2}}\left(b-\frac{t^{2} c}{1+t^{2} a}\right)^{-\frac{1}{2}}
\end{aligned}
$$

By making the substitution

$$
z=\left(\frac{t^{2} c}{1+t^{2} a}\right)^{\frac{1}{2}}
$$

the expectation value then can be written as

$$
\left\langle\phi_{k}\left|\frac{1}{r_{i j} r_{p a}}\right| \phi_{l}\right\rangle=\frac{4}{\pi} \frac{\pi^{\frac{3 n}{2}}\left|\boldsymbol{A}_{k l}\right|^{-\frac{3}{2}}}{\sqrt{c}} \int_{0}^{\sqrt{\frac{c}{a}}} \mathrm{~d} z \frac{1}{\left(b-z^{2}\right)^{\frac{1}{2}}} .
$$

Finally, we define $z=\sqrt{b} \sin (\rho)$ such that

$$
\begin{aligned}
\left\langle\phi_{k}\left|\frac{1}{r_{i j} r_{p a}}\right| \phi_{l}\right\rangle & =\frac{4}{\pi} \frac{\pi^{\frac{3 n}{2}}\left|\boldsymbol{A}_{k l}\right|^{-\frac{3}{2}}}{\sqrt{c}} \int_{0}^{\sin ^{-1}\left(\sqrt{\frac{c}{a b}}\right)} \mathrm{d} \rho \frac{\sqrt{b} \cos (\rho)}{\left(b-b \sin ^{2}(\rho)\right)^{\frac{1}{2}}} \\
& =\frac{4}{\pi} \frac{\pi^{\frac{3 n}{2}}\left|\boldsymbol{A}_{k l}\right|^{-\frac{3}{2}}}{\sqrt{c}} \int_{0}^{\sin ^{-1}\left(\sqrt{\frac{c}{a b}}\right)} \mathrm{d} \rho .
\end{aligned}
$$

Hence, evaluation of Eq. S21 leads to the final expression

$$
\left\langle\phi_{k}\left|\frac{1}{r_{i j} r_{p a}}\right| \phi_{l}\right\rangle=\frac{4}{\pi} \frac{\pi^{\frac{3 n}{2}}\left|\boldsymbol{A}_{k l}\right|^{-\frac{3}{2}}}{\sqrt{c}} \sin ^{-1}\left(\sqrt{\frac{c}{a b}}\right),
$$


for $a b-c>0, c \neq 0$.

This expression can be generalized to the $\left\langle\frac{1}{r_{i j}^{2}}\right\rangle$ expectation value that appears in the $V_{\text {ee }}^{2}$ operator of the 2-electron case. Then, the $\underline{\boldsymbol{J}}_{p a}$ matrix can be seen to be equal to the $\underline{\boldsymbol{J}}_{i j}$ matrix. The traces of Eqs. S12 S14 are such that $a=b$ and $c=a^{2}$, where we have used the fact that for a rank-1 matrix $\boldsymbol{M}$

$$
\operatorname{Tr}\left\{\boldsymbol{M}^{2}\right\}=(\operatorname{Tr}\{\boldsymbol{M}\})^{2}
$$

Equation $\mathrm{S} 22$ then reads as

$$
\left\langle\phi_{k}\left|\frac{1}{r_{i j}^{2}}\right| \phi_{l}\right\rangle=\frac{4}{\pi} \frac{\pi^{\frac{3 n}{2}}\left|\boldsymbol{A}_{k l}\right|^{-\frac{3}{2}}}{\sqrt{a^{2}}} \sin ^{-1}(1)=\frac{2 \pi^{\frac{3 n}{2}}\left|\boldsymbol{A}_{k l}\right|^{-\frac{3}{2}}}{a}
$$

\section{Fulfillment of the $x_{j}\left(\varepsilon_{1}\right) \geq \varepsilon_{j+1}$ condition}

As discussed in the paper, an essential part of the PM lower bound theory is the assurance of the lower bound property of the poles of the PM equation. For this purpose, we consider two strategies. The first one, used in Ref. 1, is to check whether $x_{j}$ decreases monotonically when increasing the dimensionality of the basis set. This test is self-contained, but not directly applicable to the current setup with ECGs. This approach is useful if the basis set is increased "systematically" without changing the already selected functions, e.g., as it is in the case of increasing the dimensionality of basis sets composed of orthogonal polynomials. The ECG basis set including functions that are generated and regularly refined based on the energy-minimization condition does not necessarily have this property.

The second strategy is testing the fulfillment of the $x_{j}\left(\varepsilon_{1}\right) \geq \varepsilon_{j+1}$ condition that requires information about ground and excited-state energies (that are generally not known). In particular, for Li, we know that we have a good upper bound from the ECG computation that can be estimated to be converged on the order of a few ppbs. Then, we may take this 
value and lower it by $50 \mathrm{ppb}$ to have a lower-bound estimate. Then, by using this value for $\varepsilon$ in the PM equation one finds the pole $x_{j}$ and checks whether it is greater than $\varepsilon_{j+1}$ (that is also taken from some other computation). Is this a "useful" check? In its present form, it requires external information that is, in general, not available. There is work underway, starting out from this particular condition that we check here, to eliminate the need to use information on state energies, if the overall computation is sufficiently accurate.

The results of this (second) test are shown for the Li atom using the energy-optimized basis set in Table $\mathrm{S} 1$. The column denoted by $x_{1}\left(\varepsilon_{1}\right)\left[\mathrm{E}_{\mathrm{h}}\right]$ shows the value of $x_{1}$ found as a function of the dimensionality of the energy-optimized ECG basis set. The last line shows the known value of the first excited state energy. One then notices that for all basis sets used, indeed $x_{1} \geq \varepsilon_{2}$ is fulfilled. Inserting a lower bound to the excited state energy, $\varepsilon_{2}^{-}$, in the PM equation gives the lower bound to the ground state energy, shown in the second column. The known converged energies are shown in the last row. The data in this column is also plotted in Fig. 1d of the paper.

Table S1: Li atom ground state energy lower bound: testing the validity of the $x_{1}\left(\varepsilon_{1}\right) \geq \varepsilon_{2}$ condition which assures that the PM expression gives a lower bound to the ground state of the Li atom when replacing $x_{1}$ with $\varepsilon_{2}$ in the PM equation. The PM matrix was calculated using the Ritz eigenvectors corresponding to an energy-optimized subspace. (See Table 2 in the paper, for the $\varepsilon_{n}$ values.)

\begin{tabular}{ccccc}
\hline \hline$L$ & $x_{0}\left(\varepsilon_{2}^{-}\right)\left[\mathrm{E}_{\mathrm{h}}\right]$ & $\lambda_{2}\left[\mathrm{E}_{\mathrm{h}}\right]$ & $x_{1}\left(\varepsilon_{1}\right)\left[\mathrm{E}_{\mathrm{h}}\right]$ \\
\hline 50 & -7.489533004 & -7.301424115 & -7.303938484 \\
100 & -7.480233615 & -7.334043730 & -7.334299893 \\
150 & -7.479359816 & -7.340602621 & -7.340681986 \\
200 & -7.478625005 & -7.344480432 & -7.344523855 \\
300 & -7.478306665 & -7.349351448 & -7.349359498 \\
400 & -7.478228894 & -7.351294035 & -7.351297365 \\
450 & -7.478278264 & -7.351489282 & -7.351490824 \\
500 & -7.478274447 & -7.351593385 & -7.351594510 \\
550 & -7.478232084 & -7.351979625 & -7.351980558 \\
650 & -7.478231080 & -7.352059482 & -7.352060145 \\
775 & -7.478184715 & -7.352827201 & -7.352827644 \\
900 & -7.478204144 & -7.352966162 & -7.352966447 \\
\hline$\varepsilon_{\text {ref }}^{2}$ & -7.478060324 & -7.354098369 & & \\
\hline \hline
\end{tabular}


Similar results are shown in Table S2 for the variance-optimized Lehmann basis set results. Here, the fourth column denoted by $x_{2}\left(\varepsilon_{1}\right)\left[\mathrm{E}_{\mathrm{h}}\right]$ is the check whether the second root of the PM equation is greater than or equal to $\varepsilon_{3}$ for which the precise value is given in the last row of the table. One notes that for all basis set sizes, $L$, the condition $x_{2}\left(\varepsilon_{1}\right) \geq \varepsilon_{3}$ is fulfilled. The last column shows the Lehmann lower bound values that are not accurate in this case.

Table S2: Li atom ground-state energy lower bound: Test for the validity of the condition $x_{2}\left(\varepsilon_{1}\right) \geq \varepsilon_{3}$. The test is shown for results based on a variance-optimized Lehmann eigenvector basis set with $\rho=\varepsilon=\varepsilon_{3}^{-}$, a lower bound to the second excited state (See Table 2 for the $\varepsilon_{n}$ values used here). Further explanation of the data is provided in the text.

\begin{tabular}{ccccc}
\hline \hline$L$ & $x_{1}\left(\varepsilon_{3}^{-}\right)\left[\mathrm{E}_{\mathrm{h}}\right]$ & $\lambda_{2}\left[\mathrm{E}_{\mathrm{h}}\right]$ & $x_{2}\left(\varepsilon_{1}\right)\left[\mathrm{E}_{\mathrm{h}}\right]$ & $\tau\left(\varepsilon_{3}^{-}\right)\left[\mathrm{E}_{\mathrm{h}}\right]$ \\
\hline 100 & -7.479975612 & -7.333411548 & -7.283362123 & -12.485954782 \\
150 & -7.494183958 & -7.338734920 & -7.032050516 & -10.219934711 \\
200 & -7.478713765 & -7.341186701 & -7.011706912 & -9.183166843 \\
300 & -7.478331289 & -7.343563794 & -7.163415099 & -8.573938957 \\
400 & -7.478183066 & -7.342453718 & -7.184330746 & -8.522021325 \\
450 & -7.478247701 & -7.343891621 & -7.098568111 & -8.497641762 \\
500 & -7.478231416 & -7.344161611 & -7.127510836 & -8.495039086 \\
550 & -7.478163737 & -7.344412016 & -7.198626209 & -8.488917246 \\
650 & -7.478144212 & -7.344961821 & -7.209320748 & -8.485418119 \\
775 & -7.478090347 & -7.345686536 & -7.281643459 & -8.477869989 \\
900 & -7.478106740 & -7.347166237 & -7.264466168 & -8.471368155 \\
\hline Ritz $^{2}$ & -7.478060324 & -7.354098369 & -7.318530665 & \\
Temple $^{3}$ & -7.478176 & & & \\
\hline \hline
\end{tabular}

Similar tests are presented for the He atom using energy optimized Lehmann eigenvector basis sets. Also in this case, the $x_{1}\left(\varepsilon_{1}\right)>\varepsilon_{2}$ condition is fulfilled in all cases. The PM lower bound to the ground state energy (second column) is superior to the Lehmann lower bound (last column), generated using the pole $\rho=\varepsilon_{2}^{-}$. 
Table S3: He atom ground state energy lower bound: Test for the validity of the condition that $x_{1}\left(\varepsilon_{1}\right) \geq \varepsilon_{2}$ for the He atom for different variance-optimized basis sets and Lehmann eigenvectors. The notation is as in Tables S1 and S2. (See Table 2 for the $\varepsilon_{n}$ values used.) The last column gives the Lehmann lower bounds.

\begin{tabular}{ccccc}
\hline \hline $\mathrm{L}$ & $x_{1}\left(\varepsilon_{2}^{-}\right)\left[\mathrm{E}_{\mathrm{h}}\right]$ & $\lambda_{2}\left[\mathrm{E}_{\mathrm{h}}\right]$ & $x_{1}\left(\varepsilon_{1}\right)\left[\mathrm{E}_{\mathrm{h}}\right]$ & $\tau\left(\varepsilon_{2}^{-}\right)\left[\mathrm{E}_{\mathrm{h}}\right]$ \\
\hline 20 & -2.913900672 & -0.701229770 & -1.658160048 & -3.163875676 \\
50 & -2.904005371 & -1.583310706 & -1.702486068 & -2.922294913 \\
100 & -2.903758953 & -1.841993018 & -1.846510861 & -2.907185738 \\
150 & -2.903731133 & -1.982594206 & -1.982751346 & -2.905210714 \\
200 & -2.903729713 & -2.004193398 & -2.004310795 & -2.904819525 \\
300 & -2.903726796 & -2.012966252 & -2.013133351 & -2.904369594 \\
400 & -2.903725983 & -2.017047976 & -2.017202116 & -2.904320053 \\
\hline$\varepsilon_{\text {ref }}$ & -2.903724377 & -2.145974046 & & \\
\hline \hline
\end{tabular}

\section{References}

(1) Pollak, E.; Martinazzo, R. Lower Bounds for Coulombic Systems. J. Chem. Theory Comput. 2021, 17, 1535-1547.

(2) King, F. W. High-Precision Calculations for the Ground and Excited States of The Lithium Atom. Adv. At., Mol., Opt. Phys. 1999, 40, 57-112.

(3) Lüchow, A.; Kleindienst, H. Accurate upper and lower bounds to the ${ }^{2} \mathrm{~S}$ states of the lithium atom. Int. J. Quantum Chem. 1994, 51, 211-224. 